\title{
Proposta de uma Interface Cérebro Computador para controle de cadeira de rodas motorizada por meio do sensor EMOTIV Insight ${ }^{\circledR}$
}

\author{
F.L.A. Silva*, E.L.M. Naves* \\ *Núcleo de Tecnologias Assistivas - Universidade Federal de Uberlândia, Uberlândia, Brasil \\ E-mail: fabricioluizalves@hotmail.com / web: www.nta.ufu.br
}

Resumo: Este trabalho objetiva inserir no contexto das tecnologias assistivas a inteligência artificial (IA), por meio do desenvolvimento de algoritmo para realização de tarefas cognitivas como, por exemplo, a execução de tarefas para tomada de decisão no controle de uma cadeira motorizada, por meio da qual o usuário com alguma deficiência motora severa (tetraplegia, esclerose lateral amiotrófica, etc.), utilize sinais evocados (BCI Brain Computer Interface). A partir de um software específico, haverá a coleta de sinais cerebrais (EEG Eletroencefalograma) do usuário, para alimentar uma rede neural, que classificará os sinais de modo a permitir o controle, em tempo real, da cadeira motorizada.

Palavras-chave: Inteligência Artificial, Rede Neural, EEG, BCI.

Abstract: This work objective to enter in the context of assistive technologies to artificial intelligence (AI), through the development of algorithm to perform cognitive tasks, such as performing tasks for decision making in the control of a motorized chair, where the user with some severe motor disability (quadriplegia, $A L S$, etc.) will use evoked signals (BCI - Brain Computer Interface). From specific software, there will be a collection of brain signals (EEG electroencephalogram) user to feed a neural network to classify the signals to allow the real-time control of the motorized chair.

Keywords: artificial intelligence, neural network, EEG, BCI.

\section{Introdução}

Dados coletados pelo IBGE mostram a prevalência dos diferentes tipos de deficiência e características das pessoas que compõem esse segmento da população. Nesse contexto, o trabalho busca disponibilizar formas para que o usuário portador de deficiência motora severa possa usufruir de mobilidade, de maneira autônoma. Para atender a esse público específico, que não realiza qualquer ação motora voluntária, o trabalho promoverá adequação ao produto que será utilizado, no caso uma cadeira motorizada, por meio da qual, por sinais evocados (BCIs - Brain Computer Interface) (Figura 1), será captado o sinal eletroencefalograma (EEG) do condutor, que será classificado e processado por uma rede neural, para realização de tarefas cognitivas [2].

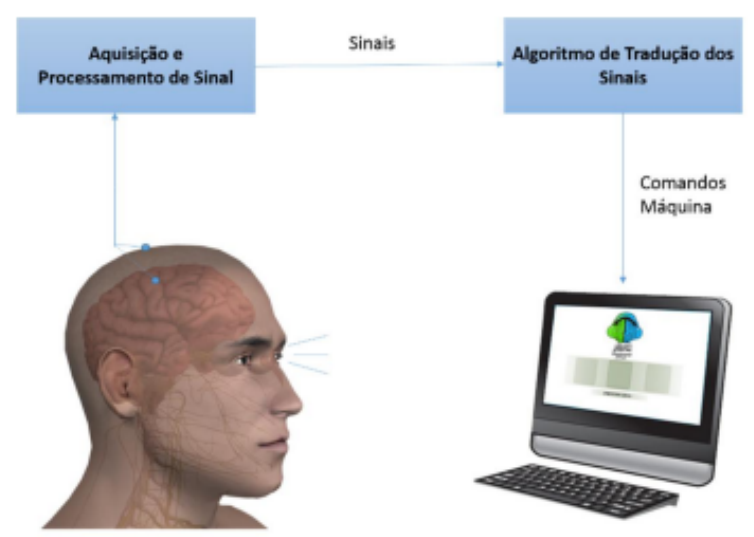

Figura 1 - Funcionamento BCI

Fonte: Wolpaw et al., 2000

O estudo com cadeiras motorizadas é feito desde o início da década de 80 e, desde então, aborda-se as mais diferentes modalidades de controle da cadeira, seja pelo piscar dos olhos, pela face e até sinais cerebrais [1]. A proposta do trabalho é apresentar novas tecnologias, como, por exemplo, oferecer ao usuário um equipamento portátil e ergonômico, com poucos eletrodos, utilizando recursos mais acessíveis para a leitura dos sinais cerebrais ou da musculatura facial, com uma interface gráfica simples, disponibilizando uma maior autonomia no uso da cadeira motorizada com mais conforto para o usuário.

\section{Materiais e métodos}

A linguagem para desenvolvimento do software se baseará no C\#, que é utilizado no controle software hardware, e utilizará banco de dados mysql para gravar o treinamento do usuário. Essa gravação se faz necessária, pois cada usuário possui características diferentes e, uma vez que a rede neural esteja treinada para aquele usuário em específico, não será necessário gravar uma segunda vez.

$\mathrm{Na}$ figura 2, apresenta-se um macro fluxo do que está sendo proposto sobre o desenvolvimento do software. 


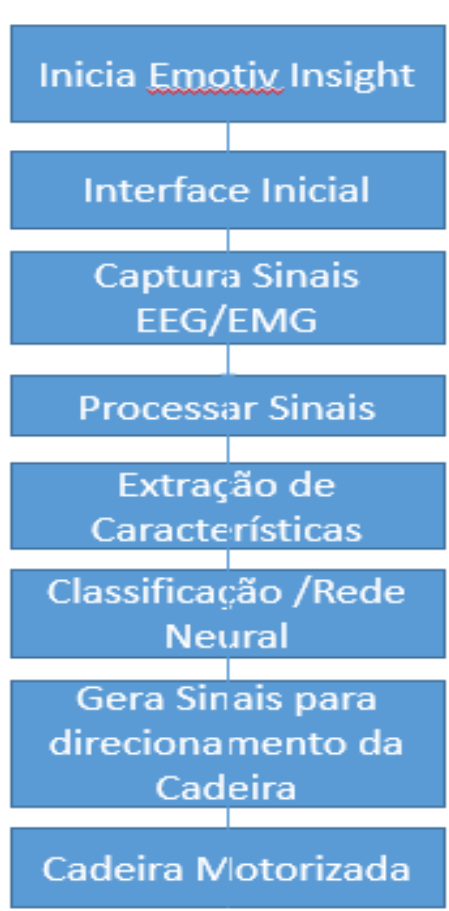

Figura 2 - Macro Fluxo

Emotiv Insight ${ }^{\circledR}$ - Aparelho desenvolvido pela empresa Emotiv, possui 5 canais, sendo: AF3, AF4, T7, $\mathrm{T} 8, \mathrm{Pz}$, com uma frequência de resposta de 0.5 a $43 \mathrm{~Hz}$. Sua conexão é feita por wireless. Conforme figura 3 :

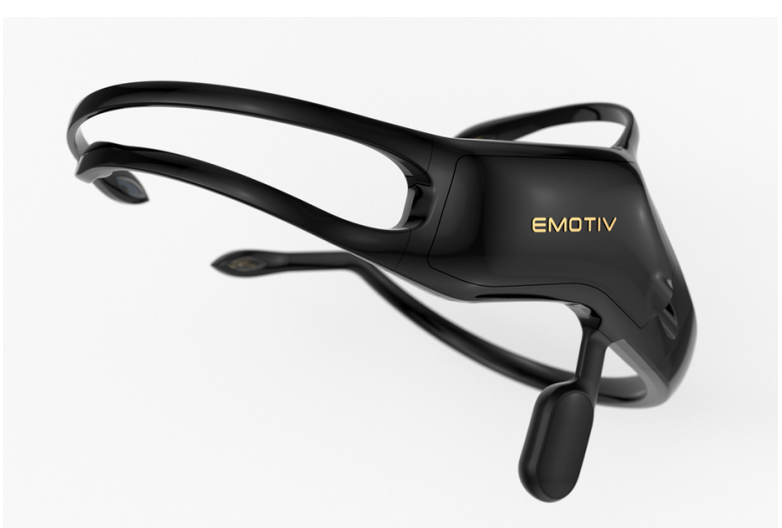

Figura 3 - Emotiv Insight $®$

Fonte: Site do Fornecedor www.emotiv.com

O Módulo de Emissão de Sinais Evocados baseia-se em uma interface cérebro-computador, visto que o método de comunicação não se dá por meio de músculos, mas de atividades cerebrais [3] e com potencial de fornecer uma ligação entre o cérebro e o mundo físico [4]. O software oferecerá setas direcionais (direita, esquerda, frente e atrás), e um quinto que simboliza parada, conforme modelo apresentado na figura 4.

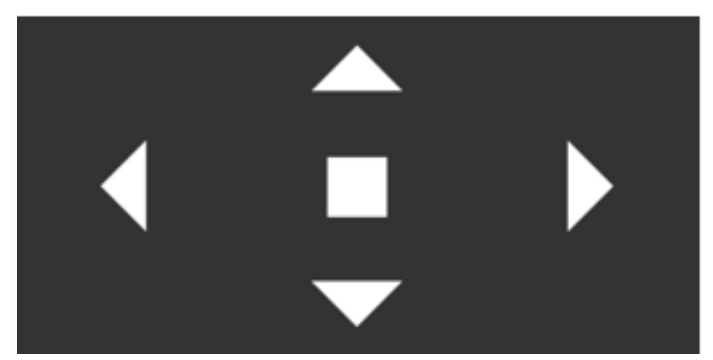

Figura 4 - Interface Protótipo do modulo

Pelos objetos (setas), serão enviados impulsos ou determinadas frequências e cada seta irá variar sua frequência entre $8-26 \mathrm{~Hz}$ [5].

Implementação do módulo de processamento dos sinais EEG (EletroencefaloGrama) - Os sinais EEG/EMG (EMG - Eletromiografia) serão enviados pelo aparelho Emotiv Insight ${ }^{\circledR}$. O processamento será feito pela análise FFT (transformada de Fourier), e com uma rede neural que será treinada com os estímulos do usuário, a fim de se extrair as características e classificação do sinal a ser utilizado em uma Rede Neural (Algoritmo Backpropagation), e representado conforme Figura 5, a mesma deverá classificar e enviar a direção por meio de valores ( 1 a 5 ) que corresponderá à ação a ser tomada pela cadeira.

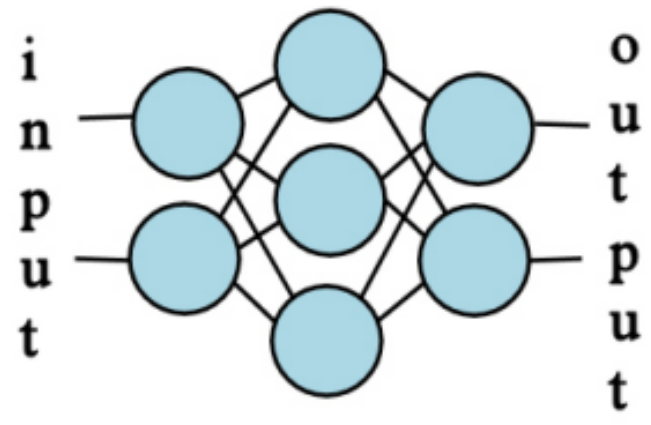

Figura 5 - Rede Neural

Testes em ambiente virtual - Os testes serão feitos utilizando o simulador virtual (VIEW) [7][8]. Nesta etapa o usuário terá um primeiro contato com os dispositivos (Softwares, Cadeira Motorizada), e será feito o cadastro e treinamento da Rede Neural. Nesse primeiro momento haverá treinamento (Usuário, Software - Figura 6), em ambiente simulando no mundo virtual.

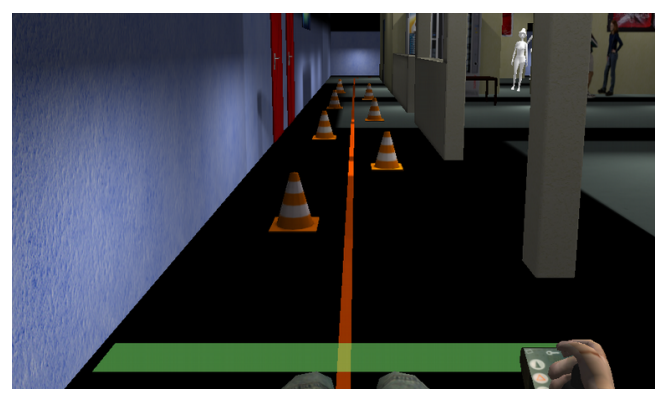


Figura 6 - Ambiente Virtual [7]

\section{Resultados}

Espera-se que a Inteligência Artificial, com todo seu potencial de processamento, classifique e apresente o resultado objetivado [8] no controle da cadeira, direcionando, de forma eficaz, de acordo com o sinal proveniente do software, todos os itens envolvidos, desde a captação dos sinais cerebrais até a classificação, e seus processamentos devem estar alinhados para que a precisão no direcionamento da cadeira seja eficaz e contribua na qualidade de vida do usuário portador de deficiência.

\section{Conclusão}

Com a demanda de sistemas reais para auxílio a pessoas com deficiências motoras severas (tetraplegia, esclerose lateral amiotrófica, etc), aparelhos como o Emotiv Insight ${ }^{\circledR}$, bem como a inteligência artificial, emergem como ferramentas promissoras ao apoio a esse nicho da população.

\section{Agradecimentos}

Os autores agradecem ao CNPq, à CAPES e à FAPEMIG pelo apoio financeiro necessário para a realização da pesquisa proposta.

\section{Referências}

[1] Bastos-Filho, Teodiano Freire, et al. "Towards a new modality-independent interface for a robotic wheelchair." IEEE transactions on neural systems and rehabilitation engineering 22.3 (2014): 567-584.

[2] Hwang, Han-Jeong, et al. "Development of an SSVEP-based BCI spelling system adopting a QWERTY-style LED keyboard." Journal of neuroscience methods 208.1 (2012): 59-65.

[3] Sanei, Saeid, and Jonathon A. Chambers. EEG signal processing. John Wiley \& Sons, 2013.

[4] Wolpaw, Jonathan R., et al. "Brain-computer interfaces for communication and control." Clinical neurophysiology 113.6 (2002): 767-791.

[5] Botti Benevides, Alessander, et al. "An Ethernet sniffer for On-line acquisition of EEG with the BrainNet36 ${ }^{\circledR}$ device applied to a BCI." Biosignals and Biorobotics Conference (2014): Biosignals and Robotics for Better and Safer Living (BRC), 5th ISSNIP-IEEE. IEEE, 2014.

[6] Morère Y, Hadj Abdelkader MA, Meliani SM, Bourhis G. Powered wheelchair driving analysis on a simulator. AAATE2011; 2011. p. 679-685.

[7] Morere Y, Bourhis G, Guilmois G, Taverne E, Coulombel L. ViEW : a simulator for the training and the evaluation of the control of an electric wheelchair. AMSE Journals, Series Modelling C, vol. 73(3); 2012. p.71-82.
[8] Norvig, Peter, and Stuart Russell. Inteligência Artificial, $3^{\text {a }}$ Edição. Vol. 1. Elsevier Brasil, 2014. 